\title{
Intraventricular Electrogram Analysis for Ventricular Tachycardia Detection: Statistical Validation
}

\author{
ROBERT D. THRONE, JANICE M. JENKINS, and LORENZO A. DiCARLO \\ From the Medical Computing Laboratory, Department of Electrical Engineering and Computer \\ Science; and the ${ }^{*}$ Cardiac Electrophysiology Laboratory, St. Joseph Mercy Hospital of the \\ Catherine McAuley Health Center, and the School of Medicine, University of Michigan, \\ Ann Arbor, Michigan
}

THRONE, R.D., ET AL.: Intraventricular Electrogram Analysis for Ventricular Tachycardia Detection: Statistical Validation. Time-domain analysis of intraventricular electrogram morphology during ventricular tachycardia (VT) and sinus rhythm or atrial fibrillation (SR/AF) has been proposed as a method for increasing the specificity of pathological tachycardia detection by antitachycardia devices. However, few studies have validated the use of such analysis with statistical methods. When statistical methods have been utilized, it has been assumed that the distribution of the values derived from analysis of the intracardiac electrograms have had a normal (gaussian) distribution. In this study, we sought to determine whether: (1) the distribution of values derived from analysis of intracardiac electrogram during SR/AF and VT is gaussian or nongaussian; and (2) the discrimination of monomorphic VT from SR/AF using SR/AF templates can be validated statistically. Two previously proposed time-domain methods-correlation waveform analysis (CWA) and area of difference (AD)-were selected for evaluation of 29 patients with 33 distinct, sustained monomorphic VTs. An initial SR/AF template was used to analyze subsequent SR/AF and VT passages with a minimum of 50 consecutive depolarizations using a "best-fit" alignment. The values derived from each analysis were examined subsequently for skewness (asymmetry) and kurtosis (shape) using two-tailed tests $(P<0.02)$. For passages of $S R / A F$, a normal (gaussian) distribution was present in only $24 \%$ (CWA), and 45\% (AD); for passages of VT, normal distribution was present in only $58 \%$ for both CWA and $A D$. Using appropriate statistical testing with nonparametric tolerance intervals, CWA and $A D$ discriminated VT from SR/AF in 29 out of $33(88 \%)$, and 30 out of $33(91 \%)$ instances, respectively, with $95 \%$ confidence. Thus, the assumption of a gaussian distribution for values derived from time-domain analysis of intraventricular electrograms for VT detection is not uniformly valid. Both CWA, which is independent of both baseline and amplitude fluctuations, and $A D$, which is not independent of these fluctuations, have similar performance when validated with appropriate statistical methods. (PACE, Vol. 13, December, Part I 1990)

statistical validation, correlation waveform analysis, area of difference, nonparametric tolerance intervals, ventricular tachycardia detection

\section{Introduction}

Although implantable devices appear to improve survival for patients whose dysrhythmias

This work was supported by NSF grant No. EET-8351215, and a Rackham Predoctoral Fellowship, University of Michigan.

Address for reprints: Lorenzo A. DiCarlo, M.D., Reichert Health Building R-3003, Catherine McAuley Health Center, P.O. Box 944 Ann Arbor, MI 48106.

Received August 23, 1990; accepted September 25, 1990. are refractory to antiarrhythmic medical therapy, present implantable devices for detection and treatment of tachycardias use simple analog circuits, and lack specificity in ventricular tachycardia (VT) recognition. ${ }^{1-7}$ Methods for detection of VT have been based primarily on timing information derived from rate. ${ }^{8-16}$ The identification of differences in intraventricular electrogram morphology during sinus rhythm (SR) and VT have been proposed to increase the accuracy of VT dis- 
crimination. ${ }^{12,17-24}$ These algorithms have usually been tested on short (10-15 seconds) passages of the dysrhythmias. ${ }^{12,18,23-26}$ Some investigators have limited their analysis to as few as 10 SR and 10 VT depolarizations. ${ }^{21}$

Few studies to date have validated the results of electrogram morphology analysis with statistical methodology, though paired Student's $t$-tests have been used in one study. ${ }^{21}$ However, paired Student's t-tests assume an underlying normal distribution of the derived similarity measures. However, the actual distribution of the values derived from time-domain methods proposed for VT detection has never been determined. Therefore, whether the results of intraventricular electrogram analysis for discriminating VT can, in fact, be appropriately validated with statistical confidence has not heretofore been demonstrated.

In this study, we analyzed two previously proposed template-matching techniques for discriminating ventricular electrograms during VT from ventricular electrograms during SR/AF: correlation waveform analysis (CWA), ${ }^{23,25,26}$ and area of difference (AD). ${ }^{20-22} \mathrm{CWA}$ is an analytic method that is independent of electrogram baseline and amplitude fluctuations, whereas $A D$ is dependent upon both fluctuations. The goals of this study were to determine whether: (1) the values derived from analyzing passages of $V T$ and SR/AF with CWA and AD have a gaussian (normal) or nongaussian distribution, and (2) VT can be distinguished with statistical certainty from SR/AF using these two different time-domain methods.

\section{Methods}

\section{Electrophysiology Study}

Bipolar $(1 \mathrm{~cm})$ distal ventricular endocardial electrograms were recorded during elective clinical cardiac electrophysiology studies as previously reported. ${ }^{23.25 .26}$ Three 6 French quadrapolar electrode catheters (USCI, Billerica, MA, USA) with an interelectrode distance of $1 \mathrm{~cm}$ were introduced and advanced under fluoroscopic guidance to the high right atrium (or right atrial appendage) and right ventricular apex. Two catheters were positioned in the right ventricular apex with one dedicated to pacing, and the other to obtaining recordings from the distal electrode pair. All recordings were made with the patients lying supine.

Ventricular electrograms were recorded on FM magnetic tape (Hewlett-Packard Models 3968 and 3964A, [San Diego, CA, USA]) from distal bipolar endocardial electrodes positioned in the right ventricular apex using amplifiers with filter settings of $0.5-500 \mathrm{~Hz}$ (Siemens Mingograf-7, Siemens-Elema, Solna, Sweden) or 1-500 Hz (PPG Biomedical Systems, Lenexa, Kansas, USA). Tape speed was $3 \frac{3}{4}$ inches per second with a bandwidth of $0-1,250 \mathrm{~Hz}$.

Data sets typically consisted of an initial passage of SR or atrial fibrillation (AF) with at least eight normal depolarizations, two or three subsequent 30-second passages during SR/AF, and a passage of monomorphic VT with at least 50 depolarizations. An SR/AF ventricular electrogram template was constructed by signal averaging eight normal ventricular depolarizations from $\mathrm{SR} / \mathrm{AF}$. This template was then compared to the ventricular electrograms in the remaining passages of SR/AF and the passage of VT using each of the template-matching techniques described below. A software trigger (peak detector) was used for the detection of waveforms. The best fit (within an $11 \mathrm{msec}$ window) alignment was used for evaluating the algorithms. ${ }^{25,26}$ All passages were digitized at $1,000 \mathrm{~Hz}$.

\section{Algorithms Analyzed}

The following notation is used in all algorithms:

$N=$ the number of points in the template.

$t_{\mathrm{i}}=$ template points.

$s_{i}=$ the signal points to be processed.

$\bar{t}=$ the template average.

$\bar{s}=$ the signal average.

$\wp=$ the value of the similarity measure.

\section{CWA}

CWA $^{23,25,26}$ uses the correlation coefficient $\rho$ as a measure of similarity between the template and waveform under analysis. The correlation coefficient is independent of both amplitude and baseline changes between the template and the signal under analysis. Mathematically the correlation coefficient is defined as: 


$$
\begin{gathered}
\rho=\frac{\sum_{i=1}^{i=N}\left(t_{i}-t\right)\left(s_{i}-s\right)}{\sqrt{\sum_{k=1}^{k=N}\left(t_{k}-\bar{t}\right)^{2}} \sqrt{\sum_{k=1}^{k=N}\left(s_{k}-s\right)^{2}}} \\
=1-\frac{1}{2} \sum_{i=1}^{i=N}\left(\frac{\left.t_{i}-\bar{t}\right)}{\sqrt{\sum_{k=1}^{k=N}\left(t_{k}-\bar{t}\right)^{2}}}-\frac{s_{i}-\bar{s}}{\sqrt{\sum_{k=1}^{k=N}\left(s_{k}-\bar{s}\right)^{2}}}\right)^{2}
\end{gathered}
$$

To avoid the square root computation, we equivalently use

$$
\wp=\operatorname{sign}(\rho) \rho^{2}
$$

where $\operatorname{sign}(\rho)$ is \pm 1 depending on the sign of $\rho$.

\section{AD}

The $\mathrm{AD}^{20-22}$ measures the absolute difference in amplitude of sample points in the template and the waveform under analysis with a similarity measure given by

$$
A D=\sum_{i=1}^{i=N}\left|t_{i}-s_{i}\right|
$$

The area of difference is usually reported as a percentage change of the absolute deviation of the template points from the baseline, i.e.,

$$
\wp=A D(\%)=\frac{\sum_{i=1}^{i=N}\left|t_{i}-s_{i}\right|}{\sum_{i=1}^{i=N}\left|t_{i}\right|} \times 100 \%
$$

\section{Testing the Hypothesis of Normality}

The data was initially examined to determine whether the hypothesis of normality of the distribution of similarity values (V) should be rejected or accepted. Two distinct tests for normality were used. First, the skewness (asymmetry) of the distribution of $V$ values during the SR/AF passages or during the VT passage was tested $(\mathrm{P}<0.02)$ using a standared two-tailed test. ${ }^{27}$ Second, the kurtosis (shape) of the distribution of $\wp$ values during the SR/AF passages or during the VT passage was tested $(P<0.02)$ using Geary's method. ${ }^{27,28}$ This was also a two-tailed test.

\section{Statistical Validation with Nonparametric Tolerance Intervals}

Statistical validation with nonparametric tolerance intervals ${ }^{27}$ assume only that the similarity values $\mathrm{V}$ are from a continuous, though unknown, probability distribution function. Therefore, it can be used to validate methods whose results produce either gaussian (normal) or nongaussian distributions.

Nonparametric tolerance intervals are constructed using the ranges of the observed values of $\mathrm{V}$ to estimate, with a given confidence level, bounds within which $\zeta \%$ of all V will occur with $95 \%$ confidence. If the intervals for VT do not overlap with the corresponding intervals for $\mathrm{SR} / \mathrm{AF}$ for a particular algorithm under analysis, the algorithm is declared to successfully separate at least $\zeta \%$ of the SR (or AF) depolarizations from at least $\zeta \%$ of the VT depolarizations with $95 \%$ confidence.

The values derived from an ideal analysis of intraventricular electrograms would separate all VT depolarizations from all SR/AF depolarizations with $100 \%$ certainty. However, this expectation may be neither realistic nor practical due to the possibility of phenomena such as sinus capture or fusion beats occurring during VT. In some cases, the local (bipolar) intraventricular electrogram wave fronts may even have characteristics which are similar when SR/AF is compared to VT.

In this study, $\zeta=90$ and 75 , bounding $90 \%$ and $75 \%$ of the values of 8 during SR /AF and VT, respectively. The minimum confidence level was set at $95 \%$.

\section{Results}

\section{Testing Hypothesis of Normality}

Tables I and II summarize the results of testing the hypothesis of normality using two-tailed tests for skewness and kurtosis $(\mathrm{P}<0.02)$. Table I summarizes the results for SR/AF, while Table II

Table I.

\begin{tabular}{lrc}
$\begin{array}{c}\text { Accept/Reject Hypothesis of } \\
\text { a Normal Distribution }\end{array}$ & CWA & AD \\
\hline Reject: Skewness alone & $6(21 \%)$ & $6(21 \%)$ \\
Reject: Kurtosis alone & $3(10 \%)$ & $2(7 \%)$ \\
Reject: Skewness and kurtosis & $13(45 \%)$ & $8(27 \%)$ \\
Accept & $7(24 \%)$ & $13(45 \%)$ \\
\hline
\end{tabular}

Summary of testing the hypothesis of an underlying normal distribution of similarity values $\wp$ during sinus rhythm or atrial fibrillation against skewness and kurtosis using a two-sided test $(P<0.02)$. There are 29 possible sinus rhythm/atrial fibrillation instances. 


\begin{tabular}{lcc}
\hline \multicolumn{3}{c}{ Table II. } \\
\hline $\begin{array}{l}\text { Accept/Reject Hypothesis of } \\
\quad \text { a Normal Distribution }\end{array}$ & CWA & AD \\
\hline Reject: Skewness alone & $7(21 \%)$ & $9(27 \%)$ \\
Reject: Kurtosis alone & $2(6 \%)$ & $3(9 \%)$ \\
Reject: Skewness and kurtosis & $5(15 \%)$ & $2(6 \%)$ \\
Accept & $19(58 \%)$ & $19(58 \%)$ \\
\hline
\end{tabular}

Summary of testing the hypothesis of an underlying normal distribution of similarity values $\$$ during monomorphic ventricular tachycardia against skewness and kurtosis using a two-sided test $(P<0.02)$. There are 33 possible ventricular tachycardia instances.

summarizes the results for VT. The top row of the tables identifies the template-matching technique used. The number of instances the normality hypothesis was rejected due to skewness, kurtosis, or both skewness and kurtosis, is summarized separately. The number of instances where the normality hypothesis was accepted is summarized at the bottom of the two tables.

For the SR/AF passages (Table I), the normality hypothesis was accepted in 7 out of $29(24 \%)$ to 13 out of $29(45 \%)$ instances, respectively, while for the VT passages (Table II) the normality hypothesis was accepted in 19 out of $33(58 \%)$ instances for both methods.

\section{Distinguishing VT from SR/AF}

Table III summarizes the results of comparing $90 \%$ and $75 \%$ tolerance intervals for CWA and AD. The first row of the table indicates the number of instances (out of 33 ) in which $75 \%$ of all SR/AF depolarizations could not be distinguished from $75 \%$ of all VT depolarizations. The second row indicates the number of instances in which at least $75 \%$ (but $<90 \%$ ) of all SR/AF depolarizations could be distinguished from at least $75 \%$ (but $<90 \%$ ) of all VT depolarizations. The final row indicates the number of instances in which at least $90 \%$ of all SR/AF could be distinguished from at least $90 \%$ of all VT depolarizations. CWA and AD could distinguish $90 \%$ of the SR/AF depolarizations from $90 \%$ of the VT depolarizations with $95 \%$ confidence in 29 out of $33(88 \%)$ and 30 out of $33(91 \%)$ instances, respectively. There were at most 2 out of $33(6 \%)$ instances that could not be distinguished when $75 \%$ of all SR/AF de- polarizations were compared to $75 \%$ of all VT depolarizations.

\section{Discussion and Conclusion}

In examining the results of CWA and AD for distinguishing ventricular electrograms during VT from those during SR/AF, the assumption that the distribution of similarity values is uniformly normal (gaussian) does not appear to be valid. Therefore, the application of statistical methods which assume an underlying normal distribution of similarity values for the purpose of validating a proposed technique for distinguishing VT from SR / AF may not be appropriate. While the use of nonparametric tolerance intervals does not require any assumption about the underlying distribution of values derived from intracardiac electrogram analysis, it is necessary that the passages under analysis be "representative" passages of both SR/AF and VT and have sufficient duration to permit valid statistical assessment.

Despite considerable differences in computational complexity, for the patient population studied and the statistical analysis method used, both of the template-matching algorithms analyzed in this study had similar statistical performance in distinguishing ventricular electrograms during SR or AF fibrillation from electrograms during VT.

The algorithms examined in this study utilized intraventricular electrograms from electrode catheters acutely placed in supine patients at rest during clinical EPS studies. Distribution analysis and statistical assessment of the effects of changes in patient position, sympathetic tone, antiarrhythmic drugs, exercise, and chronic leads is not known at present. Other time-domain methods, including those which depend upon

\begin{tabular}{|c|c|c|}
\hline$\%$ Intervals Distinguished & CWA & AD \\
\hline$<75$ & $0(0 \%)$ & $2(6 \%)$ \\
\hline$\geq 75,<90$ & $4(12 \%)$ & $1(3 \%)$ \\
\hline$\geq 90$ & $29(88 \%)$ & $30(91 \%)$ \\
\hline
\end{tabular}

Summary of separation of VT tolerance intervals from the corresponding SR/AF tolerance intervals $(95 \%$ confidence level). There are 33 VT instances. 


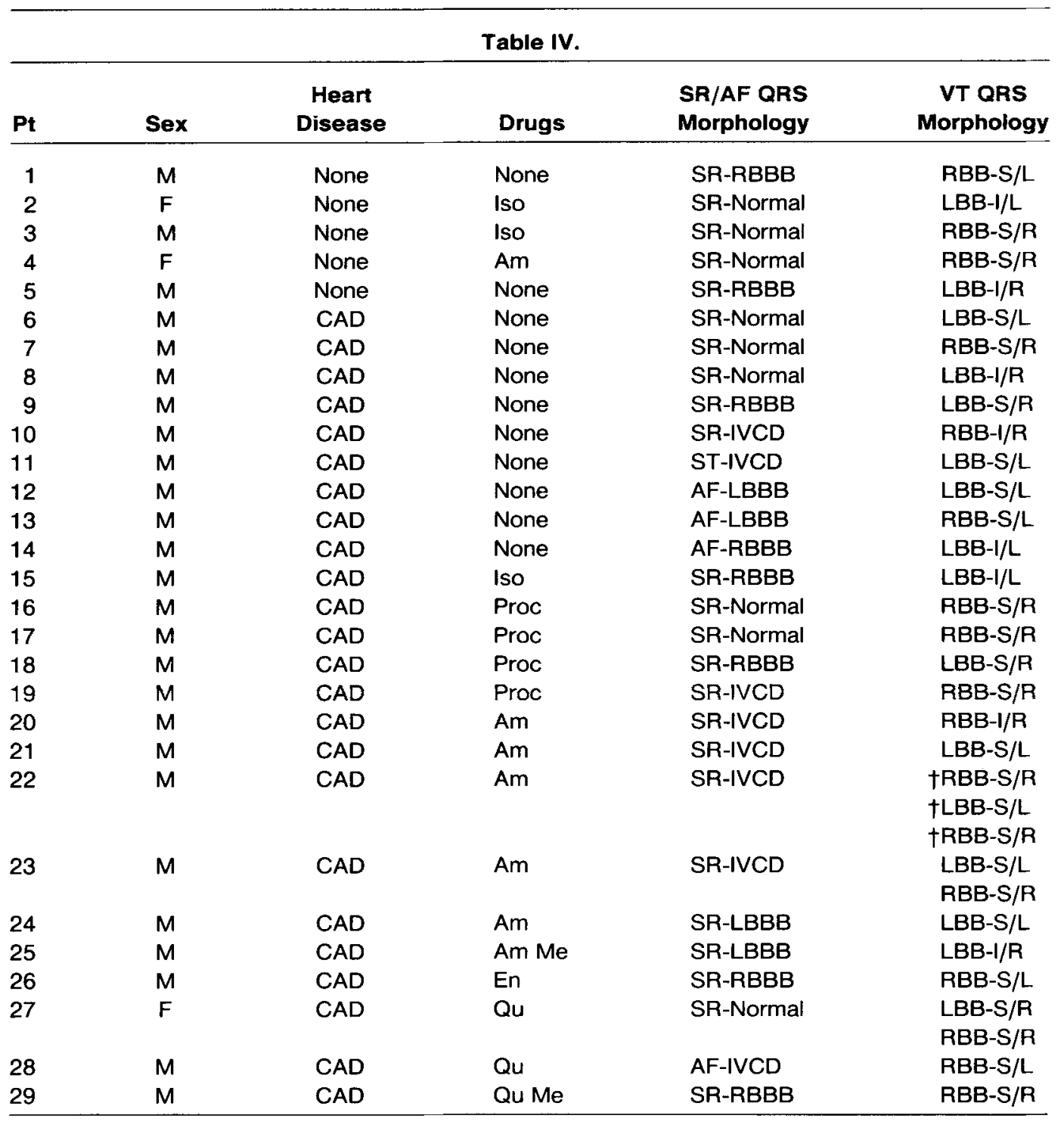

Patient data for statistical study. †These are different VT morphologies. CAD = Coronary Artery Disease; Iso $=$ Isopril, $\mathrm{Am}=$ Amiodarone, Proc $=$ Procainamide, $\mathrm{En}=$ Encainide, $\mathrm{Me}=$ Mexiletine, $\mathrm{Qu}=$ Quinidine .

electrogram baseline fluctuation alone, amplitude fluctuation alone, or which utilize other template-electrogram alignment strategies, will require similar analysis and assessment in order to validate their results with statistical confidence.
Acknowledgment: The authors wish to express their appreciation to Colleen Hoover, B.S., Barbara Nagrant, C.V.R.T., and Judy Jaffke, R.T.(R), for their technical assistance in the cardiac electrophysiology laboratory.

\section{References}

1. Echt DS, Armstrong K, Schmidt P, et al. Clinical experience, complications, and survival in 70 patients with the automatic implantable cardio-

verter/defibrillator. (abstract) Circulation 1985; 71:289.

2. Manz AS, Gerckens U, Luderitz B. Erroneous dis- 
charge from an implanted automatic cardioverter/defibrillator during supraventricular tachyarrhythmia-induced ventricular fibrillation. Am J Cardiol 1986; 7:343.

3. Platia EV, Griffith LSC, Reid PR, et al. Complications with the automatic implantable cardioverter defibrillator. (abstract) J Am Coll Cardiol 1986; 2:200.

4. Gabry MD, Brodman R, Johnston D, et al. Automatic implantable cardioverter-defibrillator: $\mathrm{Pa}$ tient survival, battery longevity, and shock delivery analysis. J Am Coll Cardiol 1987; 9:1349-1356.

5. Podczeck A, Frohner, K, Heif $\mathrm{CH}$, et al. Termination of life-threatening ventricular tachyarrhythmias by the automatic implantable cardioverter dfibrillator (AICD). (abstract) PACE 1989; 12:1256.

6. Veltri E, Mower M, Aarons D, et al. Automatic implantable cardioverter-defibrillator: Comparison of patients with and without discharge. (abstract) Circulation 1989; 80:530.

7. Steinberg JS, Sugalski JS, Haratonic K. Cardiac rhythm precipitating automatic-implantable cardioverter defibrillator discharge in outpatients: Observations from transtelephonic recordings. (abstract) Circulation 1989; 80:530.

8. Fisher JD, Goldstein M, Ostrow E, et al. Maximal rate of tachycardia development: Sinus tachycardia with sudden exercise vs. spontaneous ventricular tachycardia. PACE 1983; 6:221-228.

9. Geibel A, Zehender M, Brugada P. Changes in cycle length at the onset of sustained tachycardias-importance for antitachycardia pacing. Am Heart J 1988; 588-592.

10. Fromer M, Kus T, Dubuc M, et al. Oscillation of ventricular tachycardia cycle length. (abstract) PACE 1987; 10:451.

11. Nathan AW, Creamer JE, Davies DW, et al. Clinical experience with a new versatile, software based, tachycardia reversion pacemaker. (abstract) J Am Coll Cardiol 1986; 7:184.

12. Ripley KL, Bump TE, Arzbaecher RC. Evaluation of techniques for recognition of ventricular arrhythmias by implanted devices. IEEE Trans Biomed Eng 1989; BME-36:618-624.

13. Olson W, Bardy GH. Cycle length and morphology at the onset of spontaneous ventricular tachycardia and fibrillation. (abstract) PACE 1986; 9:284.

14. Olson W, Bardy G, Mehra R, et al. Comparison of different onset and stability algorithms for detection of spontaneous ventricular arrhythmias. (abstract) PACE 1987; 10:439.

15. Olson W, Bardy G, Mehra R, et al. Onset and stability for ventricular tachycardia detection in an implantable pacer-cardioverter-defibrillator. IEEE Computers in Cardiol 1987; 167-170.
16. Tomaselli G, Scheinman M, Griffin J. The utility of timing algorithms for distinguishing ventricular from supraventricular tachycardias. (abstract) PACE 1987; 10:415.

17. Davies DW, Wainwright RJ, Tooley MA, et al. Detection of pathological tachycardia by analysis of electrogram morphology. PACE 1986; 9:200-208.

18. Santel D, Mehra R, Olson $W H$, et al. Integrative algorithm for detection of ventricular tachyarrhythmias from the intracardiac electrogram. IEEE Computers in Cardiol 1987;175-177.

19. Pannizzo F, Furman S. Pattern recognition for tachycardia detection: A comparison of methods. (abstract) PACE 1987; 10:999.

20. Tomaselli G, Nielsen A, Finke W, et al. Morphologic differences of the endocardial electrogram in beats of sinus and ventricular origin. PACE 1988; 11:254-262.

21. Langberg J, Gibb W, Auslander D, et al. Identification of ventricular tachycardia with use of the morphology of the endocardial electrogram. Circulation 1988; 77:1363-1369.

22. Tomaselli G, Gibb W, Langberg J, et al. In vivo testing of a morphology based approach to cardiac rhythm identification. (abstract) Circulation 1987; 76:1116.

23. Lin D, DiCarlo LA, Jenkins JM. Identification of ventricular tachycardia using intracavitary ventricular electrograms: Analysis of time and frequency domain patterns. PACE 1988; 11:15921606.

24. Throne RD, Jenkins JM, Winston SA, et al. Derivative area method: A new technique for detecting ventricular tachycardia. (abstract) Circulation 1989; 80:658.

25. Throne RD, Jenkins JM, Winston SA, et al. Discrimination of retrograde from anterograde atrial activation using intracardiac electrogram waveform analysis. PACE 1989; 12:1622-1630.

26. Throne RD, DiCarlo LA, Jenkins IM, et al. Paroxysmal bundle branch block of supraventricular origin: A possible source of misdiagnosis in detecting ventricular tachycardia using time domain analyses of intraventricular electrograms. PACE 1990; 13:453-468.

27. Remington RD, Schork MA. Statistics with Applications to the Biomedical and Health Sciences, (2nd edition). Englewood Cliffs, New Jersey, Prentice-Hall, 1985.

28. Geary RC. Moments of the ratio of the mean deviation to the standard deviation for normal samples. Biometrika 1936; 28:295-307. 